\title{
Synthesis of new oxindole derivatives containing an oxazolidin-2-one
}

\author{
Abdulsalam Alsubari, ${ }^{a}$ Rachid Bouhfid, ${ }^{\text {and El Mokhtar Essassi*a,b }}$ \\ ${ }^{a}$ Laboratoire de Chimie Organique Hétérocyclique, Associé au CNRST, Pôle de compétence \\ Pharchim, Université Mohammed V-Agdal, BP. 1014 Avenue Ibn Batouta, Rabat, Maroc \\ ${ }^{b}$ Institute of Nanomaterials and Nanotechnology (INANOTECH), ENSET, \\ Av. de l'Armée Royale, Madinat El Irfane. 10100 - Rabat, Maroc \\ E-mail: emessassi@yahoo.fr
}

\begin{abstract}
The reactions of bis(2-chloroethyl)amine in presence of potassium carbonate with substituted isatins afforded the corresponding 1-[2-(2-oxo-1,3-oxazolidin-3-yl)ethyl)indoline-2,3-diones, which are used as starting materials for the preparation of new heterocyclic systems containing quinoxaline, oxazolidine, benzimidazoquinazoline and oxindole nuclei. The structures of the products were established by NMR spectroscopy, mass spectra and X-ray diffraction analysis.
\end{abstract}

Keywords: Oxindole, oxazolidine, potassium carbonate, spiro[benzimidazo[1,2-c]quinazoline], oxindole, quinoxaline

\section{Introduction}

Heterocyclic compounds containing 5- or 6-membered ring are important for their diverse biological activities. ${ }^{1}$ For example, indole-2,3-diones, which represent a large family of heterocyclic compounds, have been extensively explored for developing pharmaceutically important molecules. $N$-Substituted isatins ${ }^{2}$ especially are reported to show a wide range of biological activities such as antibacterial, ${ }^{3}$ anti-fungal ${ }^{4,5}$ antiviral $^{6}$ and anti-HIV, ${ }^{7,8}$ antileukemia. ${ }^{9}$ These compounds were also reported to have effects on central nervous system. ${ }^{10,11}$

The chemistry of oxazolidinone and its derivatives has received considerable attention owing to their synthetic and biological importance. ${ }^{12}$ The oxazolidinone moiety has been incorporated into a wide variety of therapeutically interesting compounds that have antibacterial, antifungal (Streptazoline); ${ }^{13,14}$ immunodulatory activity (Cytotoxane). ${ }^{15}$ Oxindoles containing an oxazolidinone nucleus (I and II) have been shown to have antibacterial activity and MAO-A and B inhibitory property. ${ }^{16}$ 
<smiles>COc1ccc([C@@H]2NC(=O)O[C@H]2CO)cc1</smiles>

Cytotoxane<smiles>C/C=C1\C[C@@H]2OC(=O)N3CCCC1[C@@H]2O3</smiles>

Streptazoline<smiles>COCC1CN(c2cn(C)c3cc(OCc4ccccc4)ccc23)C(=O)O1</smiles>

I<smiles>O=C1Cc2cc(N3CC(Cn4ccnn4)OC3=O)ccc2N1</smiles>

II

Figure 1

Generally, oxazolidinones aare prepared from amino alcohols, which in turn are prepared from amino acids by reduction with a metal hydride. Reaction of amino alcohols with phosgene and related compounds furnishes oxazolidinones. ${ }^{17-19}$ Also, treatment of amino alcohols with ethyl chloroformate produced carbamates which in turn can be cyclized to 2-oxazolidinones. ${ }^{20}$

In continuation of our work on the synthesis of $N$-substituted isatins, ${ }^{3,21,22}$ we herein report the synthesis and characterization of new oxindole derivatives bearing an oxazolidin-2-one sub-unit.

\section{Results and Discussion}

The alkylation of isatins 1a-b with bis(chloroethyl)amine dihydrochloride $\mathbf{2}$ at room temperature, using phase transfer catalysis conditions, yielded a one-pot synthesis of oxindoles 3a-b, containing an oxazolidinone nucleus (Scheme 1). It is worth mentioning that heating compounds $\mathbf{1 b}$ and 2 at $70{ }^{\circ} \mathrm{C}$ for 72 hours with similar reaction condition yielded compound $\mathbf{4 b}$ due to ring opening. However, heating $\mathbf{4 b}$ in presence of $5 \mathrm{~N} \mathrm{HCl}$ led to compound $\mathbf{3 b}$.

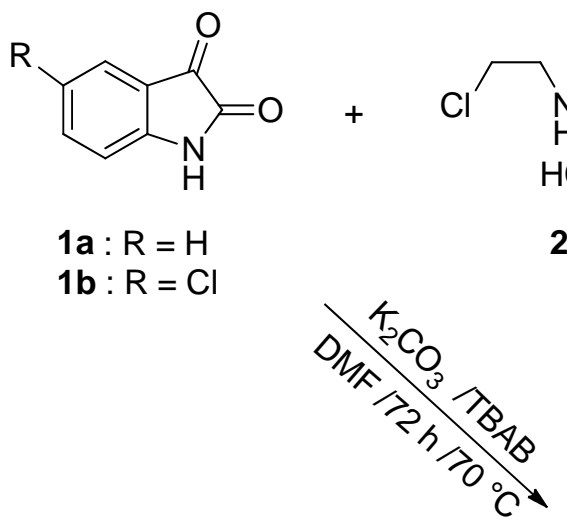

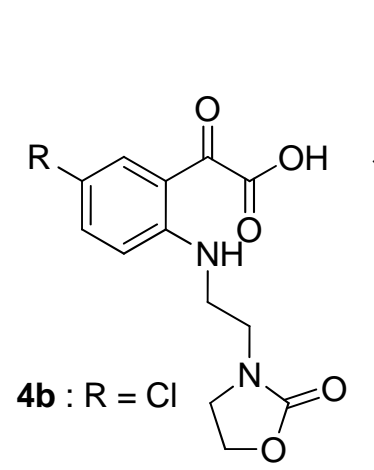

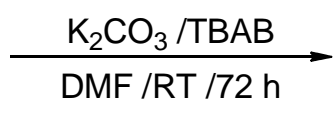

2<smiles>ClCCNCl</smiles>

\section{Scheme 1}


The structures of isolated products were confirmed by ${ }^{1} \mathrm{H},{ }^{13} \mathrm{C}$ NMR spectroscopy and mass spectrometry. For example the ${ }^{1} \mathrm{H}$ NMR spectrum of $\mathbf{3 b}$ exhibited four triplets, arising from the methylene groups $\left(\delta 3.86,3.62,3.42 \mathrm{NCH}_{2}, 4.16 \mathrm{OCH}_{2}\right)$. The aromatic protons of the indole ring system showed a multiplet in the region $(7.28-7.72 \mathrm{ppm})$. The ${ }^{13} \mathrm{C}$ NMR spectrum of $\mathbf{3 b}$, exhibited three signals at 182.6, 158.6, $158.5 \mathrm{ppm}$ for the carbonyl carbons of isatin and oxazolidine ring respectively and four signals at 37.95, 41.49, 44.54, $62.36 \mathrm{ppm}$ for the methylene groups. The mass spectrum (APCI) of $\mathbf{3 b}$ displayed the pseudo molecular ion peak at $m / z=295\left(\mathbf{M}+\mathrm{H}^{+}\right)$. A single crystal X-ray analysis of $\mathbf{3 b}$ confirmed its structure and by extrapolation, those of the analogues. An ORTEP diagram of $\mathbf{3 b}$ is shown in Figure 2.

For compound $\mathbf{4} \mathbf{b}$, the ${ }^{1} \mathrm{H}$ NMR specrum of $\mathbf{4 b}$ exhibited four triplets, arising from the methylene groups $\left(\delta 3.43,3.38,3.57 \mathrm{NCH}_{2}, 4.24 \mathrm{OCH}_{2}\right)$. The aromatic protons of the indole ring system showed a multiplet in the region $(6.85-7.56 \mathrm{ppm})$. The ${ }^{13} \mathrm{C}$ NMR spectrum of $\mathbf{4} \mathbf{b}$, exhibited three peaks at 199.9, 169.3, $158.6 \mathrm{ppm}$ for the carbonyl carbons of isatin and oxazolidine ring respectively, four peaks at 40.1, 43.2, 44.9, $62.3 \mathrm{ppm}$ for the methylene groups and $(113.7,133.8,134.3)$ for aromatic carbons. The structure of this molecule was also confirmed by single-crystal X-ray data (Figure 2).

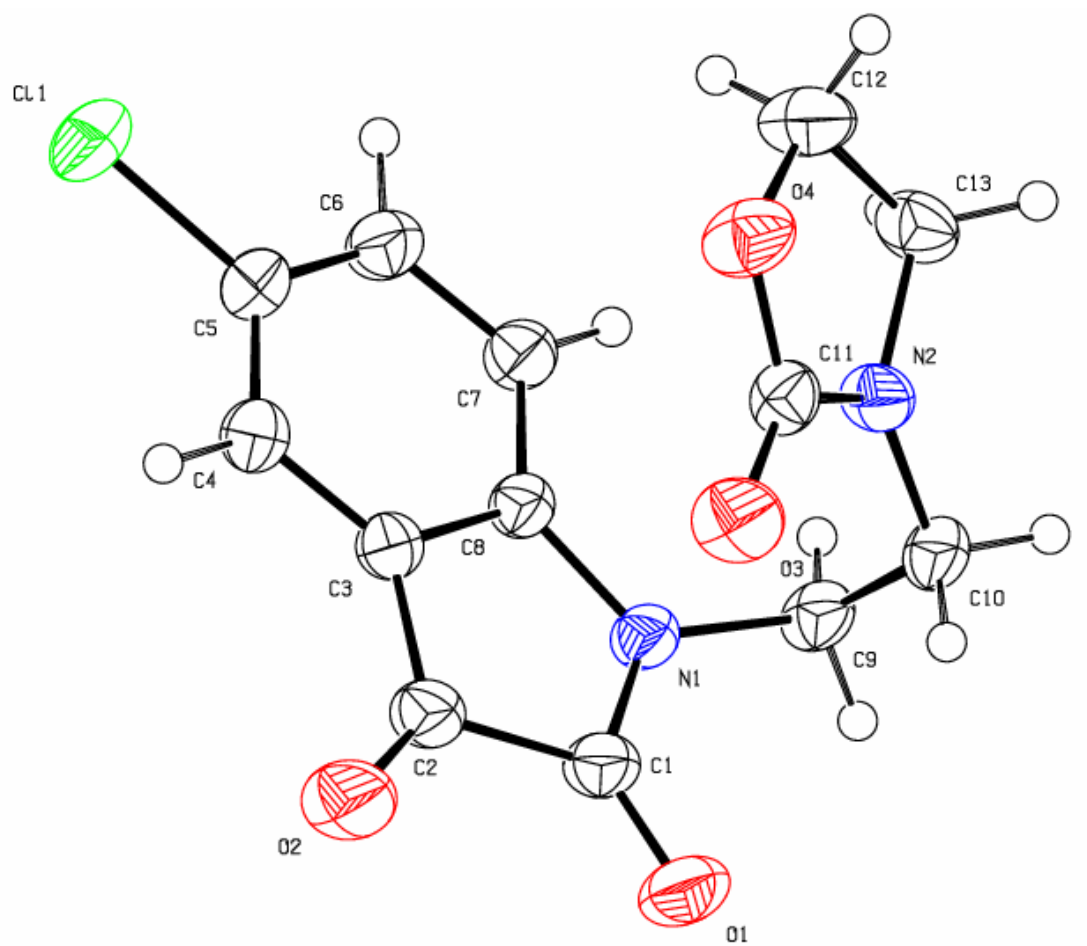




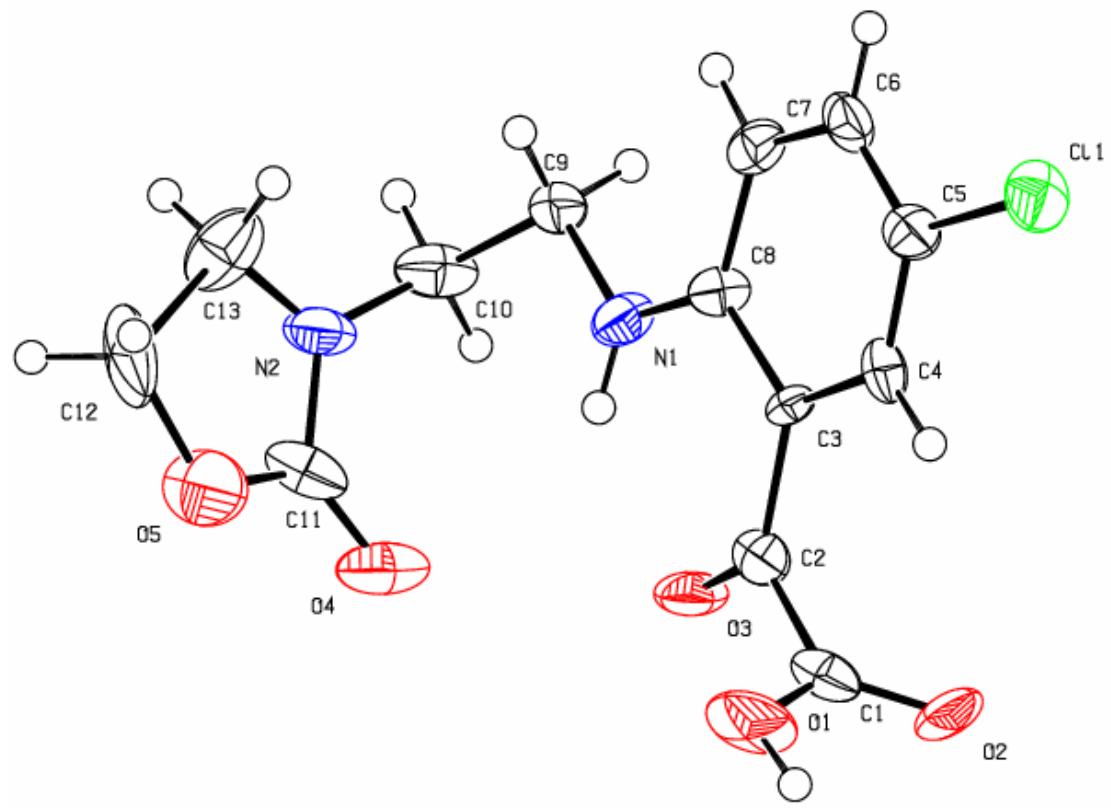

Figure 2. Molecular structure of $\mathbf{3 b}$ and $\mathbf{4 b}$, with $30 \%$ probability displacement ellipsoids.

Table 1. Crystal data and structure refinement for $3 \mathbf{b}$ and $\mathbf{4 b}$

\begin{tabular}{ccc}
\hline & 3b & 4b \\
\hline Crystal data & & \\
Formula & $\mathrm{C}_{13} \mathrm{H}_{11} \mathrm{ClN}_{2} \mathrm{O}_{4}$ & $\mathrm{C}_{13} \mathrm{H}_{13} \mathrm{ClN}_{2} \mathrm{O}_{5}$ \\
Formula Weight & 294.69 & 312.70 \\
Crystal System & triclinic & triclinic \\
Space group & $\mathrm{P}-1$ & $\mathrm{P}-1$ \\
$\mathrm{a}, \mathrm{b}, \mathrm{c}[\AA]$ & $\mathrm{a}=5.99770(10)$ & $8.9616(3)$ \\
& $\mathrm{b}=8.3572(2)$ & $13.5680(4)$ \\
& $\mathrm{c}=13.2865(2)$ & $15.0874(4)$ \\
$\alpha, \beta, \gamma\left[{ }^{\circ}\right]$ & $82.7880(10)$ & $113.126(1)$ \\
$\mathrm{V}\left[\AA^{3}\right]$ & $88.4750(10)$ & $94.855(2)$ \\
$\mathrm{Z}$ & $73.8400(10)$ & $94.104(2)$ \\
$\mathrm{D}(\mathrm{calc})\left[\mathrm{g} / \mathrm{cm}^{3}\right]$ & $634.57(2)$ & $1669.99(9)$ \\
$\mathrm{Mu}($ MoK $\alpha)\left[\mathrm{mm}^{-1}\right]$ & 4 & 4 \\
$\mathrm{~F}(000)$ & 1.542 & 0.124 \\
Temperature $(\mathrm{K})$ & 0.633 & 648 \\
Radiation $[\AA]$ & 304 & 298 \\
Data Collection & & 0.71073 \\
\hline
\end{tabular}


Table 1. Continued

\begin{tabular}{ccc}
\hline & 3b & $\mathbf{4 b}$ \\
\hline Theta Min-Max $\left[{ }^{\circ}\right]$ & $1.54,34.96$ & $1.5,30.3$ \\
Dataset & $-9: 9 ;-13: 13 ;-21: 21$ & $-12: 12 ;-18: 19 ;-21: 21$ \\
Tot., Uniq. Data, R(int) & $24424,5570,0.0287$ & $44215,9827,0.030$ \\
Refinement & & \\
Nref, Npar & 5570,188 & 9827,379 \\
R, wR2, S & $0.0420,0.1084$ & $0.0912,0.2487,1.04$ \\
Max. and Av. Shift/Error & $0.0731,0.1251$ & $1.52,0.05$ \\
Min. and Max. Resd. Dens. $\left[\mathrm{e} / \AA^{3}\right]$ & $-0.278,0.338$ & $-0.71,0.98$ \\
CCDC number ${ }^{23}$ & 691519 & 691520 \\
\hline
\end{tabular}

According to these results, we propose a mechanism for the formation of compounds 3a-b. It is postulated that initial alkylation of the nitrogen atom of the lactam functionality gave intermediates that underwent a nucleophilic reaction involving potassium carbonate. Cyclisation of these intermediates led to the formation of 3a-b (Scheme 2).

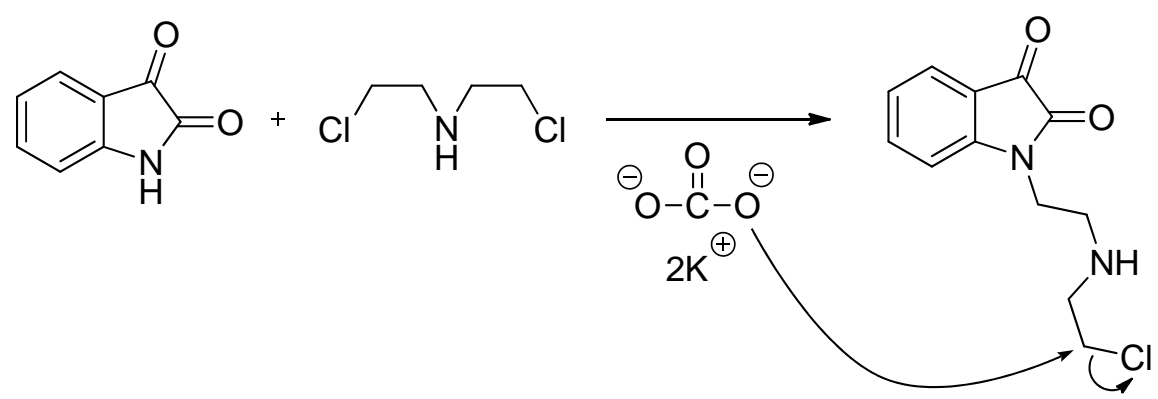<smiles>O=C1C(=O)N(CCN2CCOC2=O)c2ccccc21</smiles>

\section{Scheme 2}

In order to explore the reactivity of carbonyl group at position 3 of compounds $\mathbf{3 a}-\mathbf{b}$, we carried out the reactions of compounds 3a-b with phenylhydrazine, semicarbazide and $o$ phenylenediamine. The corresponding products: phenylhydrazones $\mathbf{5 a - b}$, semicarbazone $\mathbf{6 a}$ and indoloquinoxaline 8, were obtained in good yields (Schemes 3 and 4). 


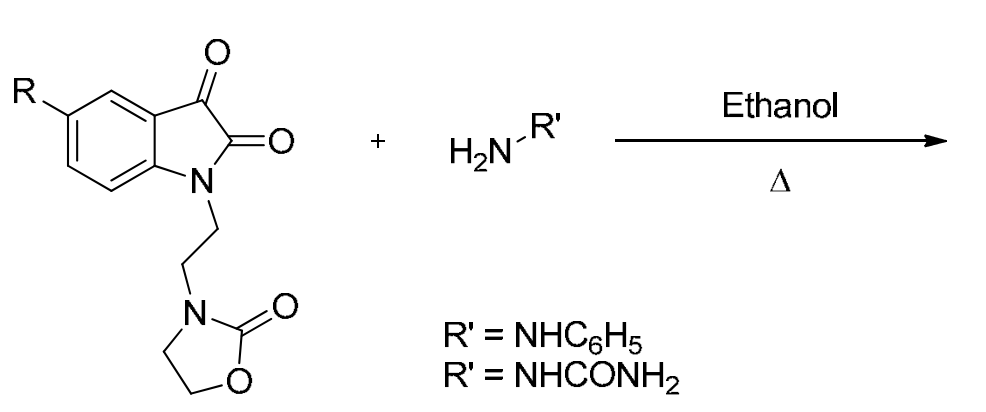<smiles>[R]N=C1C(=O)N(CCN2CCOC2=O)c2ccc([R])cc21</smiles>

$3 \mathbf{a}: \mathrm{R}=\mathrm{H}$
$\mathbf{3 b}: \mathrm{R}=\mathrm{Cl}$

\title{
Scheme 3
}
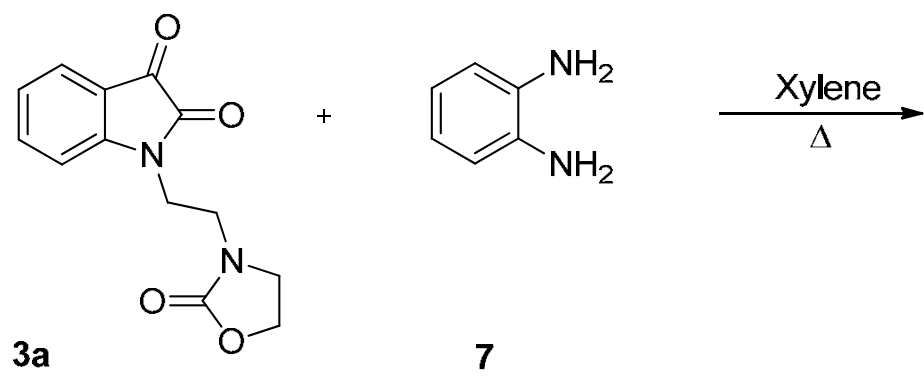

\begin{abstract}
$5 \mathbf{a}: \mathrm{R}=\mathrm{H}, \mathrm{R}^{\prime}=\mathrm{NHC}_{6} \mathrm{H}_{5}$
5b : $\mathrm{R}=\mathrm{Cl}, \mathrm{R}^{\prime}=\mathrm{NHC}_{6} \mathrm{H}_{5}$

6a: $\mathrm{R}=\mathrm{H}, \mathrm{R}^{\prime}=\mathrm{NHCONH}_{2}$
\end{abstract}

\section{Scheme 4}

The ${ }^{1} \mathrm{H}$ NMR spectrum of 5a exhibited four triplets $(\delta 3.50,3.63,3.99$ and 4.17$)$ readily recognized to arise from the methylene $\mathrm{CH}_{2}$ protons, along with multiplets $(\delta 7.06-7.70)$ for the aromatic protons. The ${ }^{13} \mathrm{C}$ NMR spectrum of 5 a showed 17 distinct resonances in agreement with the proposed structure. The carbonyl carbon resonated at $\delta 162.1 \mathrm{ppm}$ and $\mathrm{C}=\mathrm{N}$ carbon at 142.9 ppm.

Recently, studies of spiro-oxindoles have been carried out due to an increased interest in their biological activities. The oxindole ring, linked to other heterocyclic system through the spiro carbon at C-3, is of interest. In addition, various pharmacological properties are also associated with benzimidazo[1,2-c]quinazoline. ${ }^{24}$ Thus, it is possible that a benzimidazo[1,2-c]quinazoline moiety at C-3 of the oxindole, containing an oxazolidinone sub-unit, could show biological activity.

Spiro[benzimidazo[1,2-c] quinazoline-6,3'-oxindole] $\mathbf{1 0}$ was obtained by the reaction of indoline-2,3-dione derivative $3 \mathbf{b}$ with 2 -(2-aminophenyl)benzimidazole $\mathbf{9}$ in refluxing acetic acid. The structure of compound 10 was confirmed on the basis of its ${ }^{1} \mathrm{H},{ }^{13} \mathrm{C}$ NMR and mass spectral analysis. 


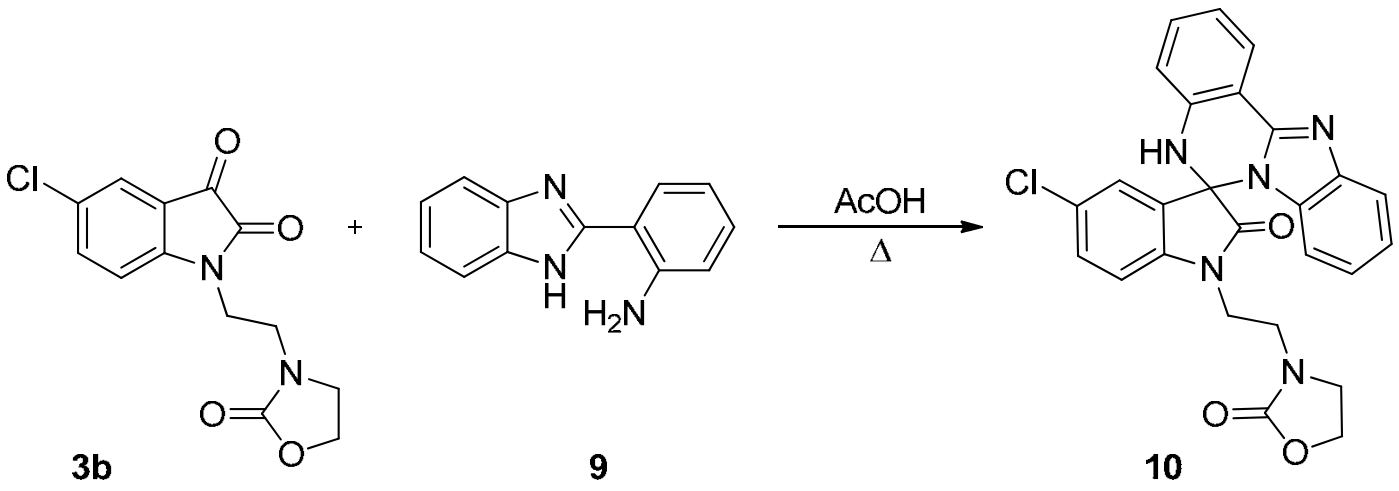

\section{Scheme 5}

The ${ }^{1} \mathrm{H}$ NMR spectrum of $\mathbf{1 0}$ shows a multiplet in the region $\delta 6.09-7.71$ for the aromatic protons. The methylene protons of the oxazolidine ring resonate as two triplets at $\delta 3.72,4.04(\mathrm{~J}$ $=7.5 \mathrm{~Hz}$ ) while the methylene protons of the alkyl chain linked to nitrogen atom of the oxindole ring appear as two triplets at $\delta 3.47,3.87(J=6 \mathrm{~Hz})$. The $\mathrm{NH}$ proton of quinazoline ring exhibits a peak at $\delta 5.63$ as a singlet. In the ${ }^{13} \mathrm{C}$ NMR spectrum of $\mathbf{1 0}$ the signals were observed at $\delta 73.3$ (spiro carbon), 38.1, 41.4, 44.6, 62.2 (methylene carbons), 158.7, 172.1 (both $\mathrm{C}=\mathrm{O}$ ).

\section{Conclusions}

In summary, we have successfully synthesized isatin derivatives bearing oxazolidin-2-one rings.

\section{Experimental Section}

General. Melting points were determined in one-end-open capillary tubes on a Büchi melting point apparatus and are uncorrected. ${ }^{1} \mathrm{H}$ NMR, ${ }^{13} \mathrm{C}$ NMR spectra were recorded on a Bruker Avance $(300 \mathrm{MHz})$ Spectrometer. Chemical shifts are reported in parts per million (ppm) using tetramethyl silane (TMS) as the internal standard, multiplicities were determined by the DEPT 135 sequence. Coupling constants were reported in Hertz (Hz). Splitting patterns were designated as s: singlet; $d$ : doublet, t: triplet. Mass spectra were recorded on VARIAN MAT 311A Spectrometer.

1-(2-(2-Oxooxazolidin-3-yl)ethyl)indoline-2,3-dione 3a. To a stirred mixture of isatin 1a (6.8 $\mathrm{mmol})$ and $\mathrm{K}_{2} \mathrm{CO}_{3}(8.16 \mathrm{mmol})$ in dimethylformamide $(30 \mathrm{~mL})$ at room temperature, was added tetra- $n$-butylammonium bromide $(0.1 \mathrm{mmol})$ and bis(chloroethyl)amine dihydrochloride $(8.16$ mmol). The mixture was stirred at room temperature for $72 \mathrm{~h}$. The white solid formed was filtered off and the solvent was evaporated under vacuum and the remaining foam was dissolved 
in $\mathrm{CH}_{2} \mathrm{Cl}_{2}$ and filtered. The $\mathrm{CH}_{2} \mathrm{Cl}_{2}$ was removed and the residue was recrystallized in ethanol to offer the pure product. Mp: $131{ }^{\circ} \mathrm{C}$. Yield: $60 \%$. ${ }^{1} \mathrm{H}$ NMR $\left(300 \mathrm{MHz}, \mathrm{CDCl}_{3}\right): \delta 3.56(\mathrm{t}, 2 \mathrm{H}$, $\mathrm{NCH}_{2},{ }^{3} J=6.0 \mathrm{~Hz}$ ); 3.70 (t, 2H, $\left.\mathrm{NCH}_{2},{ }^{3} J=7.5 \mathrm{~Hz}\right) ; 3.92\left(\mathrm{t}, 2 \mathrm{H}, \mathrm{NCH}_{2},{ }^{3} J=6.0 \mathrm{~Hz}\right) ; 4.26(\mathrm{t}, 2 \mathrm{H}$, $\left.\mathrm{OCH}_{2},{ }^{3} \mathrm{~J}=7.5 \mathrm{~Hz}\right) ; 7.02-7.62\left(\mathrm{~m}, 4 \mathrm{H}, \mathrm{H}_{\mathrm{Ar}}\right) .{ }^{13} \mathrm{C} \mathrm{NMR}\left(75 \mathrm{MHz}, \mathrm{CDCl}_{3}\right): \delta 37.7,41.7,45.0$ $3\left(\mathrm{NCH}_{2}\right) ; 62.1 \quad\left(\mathrm{OCH}_{2}\right) ; 110.1,124.1,125.6,138.6 \quad\left(\mathrm{CH}_{\mathrm{Ar}}\right) ; 182.7 \quad\left(\mathrm{C}=\mathrm{O}_{\text {ketone }}\right) ; 158.6$ (2) $\left(\mathrm{C}=\mathrm{O}_{\text {amide }}\right) ; 150.3(\mathrm{O}-\mathrm{C}=\mathrm{O}) 117.7(\mathrm{Cq}-\mathrm{N})$; Mass spectra $\left(\mathrm{CI},\left[\mathrm{MH}^{+}\right] \mathrm{m} / z\right): 261$. Anal. Calcd for $\mathrm{C}_{13} \mathrm{H}_{12} \mathrm{~N}_{2} \mathrm{O}_{4}$ : C, 60.00; H, 4.65; N, 10.76\% Found: C, 60.07; H, 4.55; N, 10.70\%.

5-Chloro-1-(2-(2-oxooxazolidin-3-yl)ethyl)indoline-2,3-dione 3b. Preparation as described for 3a starting from 1b. Mp: $194{ }^{\circ} \mathrm{C}$. Yield: 65\%. ${ }^{1} \mathrm{H}$ NMR $\left(300 \mathrm{MHz}, \mathrm{DMSO}-d_{6}\right): \delta=3.42(\mathrm{t}, 2 \mathrm{H}$, $\left.\mathrm{NCH}_{2},{ }^{3} \mathrm{~J}=6 \mathrm{~Hz}\right) ; 3.62\left(\mathrm{t}, 2 \mathrm{H}, \mathrm{NCH}^{2},{ }^{3} \mathrm{~J}=7.5 \mathrm{~Hz}\right) ; 3.86\left(\mathrm{t}, 2 \mathrm{H}, \mathrm{NCH}_{2},{ }^{3} \mathrm{~J}=6 \mathrm{~Hz}\right) ; 4.16(\mathrm{t}, 2 \mathrm{H}$, $\left.\mathrm{OCH}_{2},{ }^{3} J=7.5 \mathrm{~Hz}\right) ; 7.28-7.72\left(\mathrm{~m}, 3 \mathrm{H}, \mathrm{H}_{\mathrm{Ar}}\right) .{ }^{13} \mathrm{C} \mathrm{NMR}\left(75 \mathrm{MHz}, \mathrm{DMSO}-d_{6}\right): \delta=62.4\left(\mathrm{OCH}_{2}\right)$; 38.0, 41.5, $44.5\left(\mathrm{NCH}_{2}\right) ; 112.8,124.5,137.7\left(\mathrm{CH}_{\mathrm{Ar}}\right) ; 119.2,128.1,149.6(\mathrm{Cq}) ; 158.5$ $\left(\mathrm{C}=\mathrm{O}_{\text {oxazolidine }}\right) ; 158.6\left(\mathrm{C}=\mathrm{O}_{\text {amide }}\right) ; 182.6\left(\mathrm{C}=\mathrm{O}_{\text {ketone }}\right)$; Mass spectra $\left(\mathrm{CI},\left[\mathrm{MH}^{+}\right] \mathrm{m} / \mathrm{z}\right.$ : 295). Anal. Calcd for $\mathrm{C}_{13} \mathrm{H}_{11} \mathrm{ClN}_{2} \mathrm{O}_{4}$ : C, 52.98; H, 3.76; N, 9.51\% Found: C, 53.05; H, 3.83; N, 9.45\%.

2-(5-Chloro-2-(2-(2-oxooxazolidin-3-yl)ethylamino)phenyl)-2-oxoacetic acid $\mathbf{4 b}$. Similar to 3a, the mixture was heated at $70{ }^{\circ} \mathrm{C}$ for $72 \mathrm{~h}$. Mp: $96{ }^{\circ} \mathrm{C}$. Yield: $20 \%$. ${ }^{1} \mathrm{H}$ NMR $(300 \mathrm{MHz}$, $\left.\mathrm{CDCl}_{3}\right): \delta=4.26\left(\mathrm{t}, 2 \mathrm{H}, \mathrm{OCH}_{2},{ }^{3} \mathrm{~J}=1.12 \mathrm{~Hz}\right) ; 3.57\left(\mathrm{t}, 2 \mathrm{H}, \mathrm{NHCH}_{2},{ }^{3} \mathrm{~J}=1.66 \mathrm{~Hz}\right) ; 3.44,3.36(2 \mathrm{xt}$, $\left.4 \mathrm{H}, \mathrm{CH}_{2}-\mathrm{N}-\mathrm{CH}_{2}\right) ; 9.98\left(1 \mathrm{H}, \mathrm{CO}_{2} \mathrm{H}\right) ; 8.62(1 \mathrm{H}, \mathrm{NH}) ; 6.82-7.57\left(\mathrm{~m}, 4 \mathrm{H}, \mathrm{H}_{\mathrm{Ar}}\right) .{ }^{13} \mathrm{C}-\mathrm{NMR}(75 \mathrm{MHz}$, $\left.\mathrm{CDCl}_{3}\right): \delta=62.8\left(\mathrm{OCH}_{2}\right) ; 38.2,41.5\left(\mathrm{CH}_{2} \mathrm{NCH}_{2}\right) ; 43.6\left(\mathrm{NHCH}_{2}\right) ; 112.4,124, \mathrm{O} 8125,56,136.62$ $\left(\mathrm{CH}_{\mathrm{Ar}}\right) ; 199.9\left(\mathrm{C}=\mathrm{O}_{\text {ketone }}\right) ; 168.2\left(\mathrm{C}=\mathrm{O}_{\text {amide }}\right) ; 157.4\left(\mathrm{CO}_{2} \mathrm{H}\right) ; 153.2(\mathrm{Cq}-\mathrm{NH}) ; 138.9(\mathrm{Cq}-\mathrm{Cl})$; $116.5(\mathrm{Cq}-\mathrm{C}=\mathrm{O})$. Mass spectra $\left(\mathrm{CI},\left[\mathrm{MH}^{+}\right] \mathrm{m} / z\right)$ : 313. Anal. Calcd for $\mathrm{C}_{13} \mathrm{H}_{13} \mathrm{ClN}_{2} \mathrm{O}_{5}: \mathrm{C}, 49.93$; H, 4.19; N, 8.96\% Found: C, 50.01; H, 4.11; N, 9.07\%.

3-(2-(2-Oxo-3-(2-phenylhydrazono)indolin-1-yl)ethyl)oxazolidin-2-one 5a. A mixture of isatin $(1.00 \mathrm{~g}, 6.8 \mathrm{mmol})$ and aniline $(0.62 \mathrm{~mL}, 6.8 \mathrm{mmol})$ in absolute ethanol $(20 \mathrm{~mL})$ was heated at reflux for $5 \mathrm{~h}$. The reaction mixture was allowed to cool and the resulting precipitate filtered from the solution. The crude product was recrystallized in ethanol. Mp: $192{ }^{\circ} \mathrm{C}$. Yield: 89\%. ${ }^{1} \mathrm{H}$ NMR (300 MHz, $\left.\mathrm{CDCl}_{3}\right): \delta 3.50\left(\mathrm{t}, 2 \mathrm{H}, \mathrm{NCH}_{2},{ }^{3} \mathrm{~J}=6 \mathrm{~Hz}\right) ; 3.63\left(\mathrm{t}, 2 \mathrm{H}, \mathrm{NCH}_{2},{ }^{3} J=7.2\right.$ $\mathrm{Hz}) ; 3.99$ (t, 2H, $\left.\mathrm{NCH}_{2},{ }^{3} \mathrm{~J}=6 \mathrm{~Hz}\right) ; 4.17$ (t, 2H, $\left.\mathrm{OCH}_{2},{ }^{3} \mathrm{~J}=7.2 \mathrm{~Hz}\right) ; 7.06-7.70\left(\mathrm{~m}, 9 \mathrm{H}, \mathrm{H}_{\mathrm{Ar}}\right) .{ }^{13} \mathrm{C}$ NMR (75 MHz, $\left.\mathrm{CDCl}_{3}\right): \delta 37.4,42.1,44.73\left(\mathrm{NCH}_{2}\right) ; 62.3\left(\mathrm{OCH}_{2}\right) ; 109.5,2 \times 114.7,118.9$, 122.8, 123.6, 128.8, 2×129.9 $\left(\mathrm{CH}_{\mathrm{Ar}}\right) ; 121.1,127.3,140.7(\mathrm{Cq}) ; 142.9(\mathrm{C}=\mathrm{N}) ; 158.3,162.1$ $\left(\mathrm{C}=\mathrm{O}_{\text {amide }}\right)$;. Mass spectra $\left(\mathrm{CI},\left[\mathrm{MH}^{+}\right] \mathrm{m} / \mathrm{z}\right)$ : 351. Anal. Calcd for $\mathrm{C}_{19} \mathrm{H}_{18} \mathrm{~N}_{4} \mathrm{O}_{3}: \mathrm{C}, 65.13 ; \mathrm{H}, 5.18$; $\mathrm{N}, 15.99$. Found : C, 65.05; H, 5.25; N, 16.07\%.

3-(2-(5-Chloro-2-oxo-3-(2-phenylhydrazono)indolin-1-yl)ethyl)oxazolidin-2-one $5 \mathbf{b}$. This compound was made using the method for 5a. Mp : $210{ }^{\circ} \mathrm{C}$. Yield: $84 \%$. ${ }^{1} \mathrm{H} \mathrm{NMR} \mathrm{(300} \mathrm{MHz,}$ $\left.\mathrm{CDCl}_{3}\right): \delta 3.48\left(\mathrm{t}, 2 \mathrm{H}, \mathrm{NCH}_{2},{ }^{3} J=6 \mathrm{~Hz}\right) ; 3.63\left(\mathrm{t}, 2 \mathrm{H}, \mathrm{NCH}_{2},{ }^{3} J=7.2 \mathrm{~Hz}\right) ; 3.98\left(\mathrm{t}, 2 \mathrm{H}, \mathrm{NCH}_{2},{ }^{3} J=\right.$ $6 \mathrm{~Hz}) ; 4.17\left(\mathrm{t}, 2 \mathrm{H}, \mathrm{OCH}_{2},{ }^{3} \mathrm{~J}=7.2 \mathrm{~Hz}\right) ; 7.09-7.60\left(\mathrm{~m}, 8 \mathrm{H}, \mathrm{H}_{\mathrm{Ar}}\right) .{ }^{13} \mathrm{C} \mathrm{NMR}\left(75 \mathrm{MHz}, \mathrm{CDCl}_{3}\right): \delta$ $37.4,42.0,44.63 \times\left(\mathrm{NCH}_{2}\right) ; 62.3\left(\mathrm{OCH}_{2}\right) ; 111.1,2 \times 115.1,115.1,118.5,124.1,128.1,2 \times 129.9$ $\left(\mathrm{CH}_{\mathrm{Ar}}\right) ; 122.8,125.9,127.2,139.2,(\mathrm{Cq}) ; 142.7(\mathrm{C}=\mathrm{N}) ; 158.3,161.7\left(\mathrm{C}=\mathrm{O}_{\text {amide }}\right)$. Mass spectra $\left(\mathrm{CI},\left[\mathrm{MH}^{+}\right] \mathrm{m} / z\right)$ : 385. Anal. Calcd for $\mathrm{C}_{19} \mathrm{H}_{17} \mathrm{ClN}_{4} \mathrm{O}_{3}: \mathrm{C}, 59.30 ; \mathrm{H}, 4.45 ; \mathrm{N}, 14.56 \%$ Found: $\mathrm{C}$, $59.41 ; \mathrm{H}, 4.33 ; \mathrm{N}, 14.59 \%$. 
2-(2-Oxo-1-(2-(2-oxooxazolidin-3-yl)ethyl)indolin-3-ylidene)hydrazinecarboxamide 6a. This compound was made using the method for 5a. Mp : $163{ }^{\circ} \mathrm{C}$. Yield: $86 \%$. ${ }^{1} \mathrm{H}$ NMR $(300 \mathrm{MHz}$, $\left.\mathrm{CDCl}_{3}\right): \delta 3.47\left(\mathrm{t}, 2 \mathrm{H}, \mathrm{NCH}_{2},{ }^{3} J=6 \mathrm{~Hz}\right) ; 3.63\left(\mathrm{t}, 2 \mathrm{H}, \mathrm{NCH}_{2},{ }^{3} J=7.2 \mathrm{~Hz}\right) ; 3.94\left(\mathrm{t}, 2 \mathrm{H}, \mathrm{NCH}_{2},{ }^{3} J\right.$ $=6 \mathrm{~Hz}) ; 4.16\left(\mathrm{t}, 2 \mathrm{H}, \mathrm{OCH}_{2},{ }^{3} \mathrm{~J}=7.2 \mathrm{~Hz}\right) ; 7.10-7.66\left(\mathrm{~m}, 4 \mathrm{H}, \mathrm{H}_{\mathrm{Ar}}\right) .{ }^{13} \mathrm{C} \mathrm{NMR}\left(75 \mathrm{MHz}, \mathrm{CDCl}_{3}\right): \delta$ 37.4, 41.8, $44.53 \times\left(\mathrm{NCH}_{2}\right) ; 62.3\left(\mathrm{OCH}_{2}\right) ; 109.9,120.9,123.2,130.7\left(\mathrm{CH}_{\mathrm{Ar}}\right) ; 120.2,130.4(\mathrm{Cq})$; $141.4(\mathrm{C}=\mathrm{N}) ; 155.3,158.4,161.6\left(\mathrm{C}=\mathrm{O}_{\text {amide }}\right)$. Mass spectra $\left(\mathrm{CI},\left[\mathrm{MH}^{+}\right] \mathrm{m} / z\right): 318$. Anal. Calcd for $\mathrm{C}_{14} \mathrm{H}_{15} \mathrm{~N}_{5} \mathrm{O}_{4}$ : C, 52.99; H, 4.76; N, 22.07\% Found: C, 53.10; H, 4.69; N, 22.15\%.

3-(2-(6H-Indolo[2,3-b]quinoxalin-6-yl)ethyl)oxazolidin-2-one 8. A mixture of oxindole 3a (0.5 g, 3.84 mmole) and $o$-phenylenediamine $7(0.41 \mathrm{~g}, 3.84 \mathrm{mmole})$ in xylene $(30 \mathrm{~mL})$ was refluxed for $12 \mathrm{~h}$. The reaction mixture was evaporated under reduce pressure. The residue was recrystallized in ethanol. Mp : $202{ }^{\circ} \mathrm{C}$. Yield: $62 \% .{ }^{1} \mathrm{H}$ NMR $\left(300 \mathrm{MHz}, \mathrm{CDCl}_{3}\right): \delta 3.63(\mathrm{t}, 2 \mathrm{H}$,

$\left.\mathrm{NCH}_{2},{ }^{3} \mathrm{~J}=6 \mathrm{~Hz}\right) ; 3.74$ (t, 2H, $\mathrm{NCH}_{2},{ }^{3} \mathrm{~J}=7.2 \mathrm{~Hz}$ ); 4.02 (t, $\left.2 \mathrm{H}, \mathrm{NCH}_{2},{ }^{3} J=6 \mathrm{~Hz}\right) ; 4.62$ (t, 2H, $\left.\mathrm{OCH}_{2},{ }^{3} \mathrm{~J}=7.2 \mathrm{~Hz}\right) ; 7.39-8.40\left(\mathrm{~m}, 8 \mathrm{H}, \mathrm{H}_{\mathrm{Ar}}\right) .{ }^{13} \mathrm{C} \mathrm{NMR}\left(75 \mathrm{MHz}, \mathrm{CDCl}_{3}\right): \delta 39.2,42.8,44.5$ $3 \times\left(\mathrm{NCH}_{2}\right) ; 62.1\left(\mathrm{OCH}_{2}\right) ; 110.5,121.5,122.6,126.5,127.9,129.4,129.5,131.8\left(\mathrm{CH}_{\mathrm{Ar}}\right) ; 119.2$, 139.2, 140.1, $140.3(\mathrm{Cq}) ; 144.7,145.9(\mathrm{C}=\mathrm{N}) ; 158.4\left(\mathrm{C}=\mathrm{O}_{\text {amide }}\right)$. Mass spectra $\left(\mathrm{CI},\left[\mathrm{MH}^{+}\right] \mathrm{m} / \mathrm{z}\right)$ : 333. Anal. Calcd for $\mathrm{C}_{19} \mathrm{H}_{16} \mathrm{~N}_{4} \mathrm{O}_{2}$ : C, 68.66; H, 4.85; N, 16.86\% Found: C, 68.75; H, 4.78; N, $16.94 \%$.

Spiro[benzimidazo[1,2-c]quinazoline-6,3'-oxindole] 10. A mixture of oxindole $3 b$ (3.84 mmole) and 2-(2-aminophenyl)benzimidazole 9 (3.84 mmole) in acetic acid (30 $\mathrm{mL})$ was refluxed for $12 \mathrm{~h}$. The reaction mixture was evaporated under reduce pressure. The residue was recrystallized in methylenechloride. $\mathrm{Mp}>300{ }^{\circ} \mathrm{C}$. Yield: $85 \% .{ }^{1} \mathrm{H}$ NMR $\left(300 \mathrm{MHz}, \mathrm{DMSO}-\mathrm{d}_{6}\right)$ : $\delta=3.47\left(\mathrm{t}, 2 \mathrm{H}, \mathrm{NCH}_{2},{ }^{3} J=6 \mathrm{~Hz}\right) ; 3.72\left(\mathrm{t}, 2 \mathrm{H}, \mathrm{NCH}_{2},{ }^{3} J=7.5 \mathrm{~Hz}\right) ; 3.87\left(\mathrm{t}, 2 \mathrm{H}, \mathrm{NCH}_{2},{ }^{3} J=6\right.$ $\mathrm{Hz}) ; 4.04\left(\mathrm{t}, 2 \mathrm{H}, \mathrm{OCH}_{2},{ }^{3} J=7.5 \mathrm{~Hz}\right) ; 6.09-8.05\left(\mathrm{~m}, 3 \mathrm{H}, \mathrm{H}_{\mathrm{Ar}}\right) ;{ }^{13} \mathrm{C} \mathrm{NMR}\left(75 \mathrm{MHz}, \mathrm{DMSO}-\mathrm{d}_{6}\right): \delta$ $=38.1$, 41.4, 44.6, $62.2\left(\mathrm{CH}_{2}\right)$; $73.3\left(\mathrm{C}_{\text {spiro }}\right)$; 110.1, 112.4, 115.1, 119.6, 123.2, 123.4, 125.1, 126.6, 132.1, $132.7\left(\mathrm{CH}_{\mathrm{Ar}}\right)$; 112.1, 127.9, 128.5, 142.2, 142.4, 144.5, 148.2, 158.7, 172.1 (Cq). Mass spectra (CI, $\left.\left[\mathrm{MH}^{+}\right] \mathrm{m} / \mathrm{z}\right): 486$. Anal. Calcd for $\mathrm{C}_{26} \mathrm{H}_{20} \mathrm{ClN}_{5} \mathrm{O}_{3}: \mathrm{C}, 64.27 ; \mathrm{H}, 4.15 ; \mathrm{N}, 14.41 \%$ Found: C, 64.33; H, 4.19; N, 14.29\%.

\section{Acknowledgements}

The authors would like to acknowledge the financial support provided by the ministry ESRSFC of the Moroccan Government (Pôle de Compétences Pharmacochimie) and the ministry ESRS of the Yemen.

\section{References and Notes}

1. Chevan, P.; Mane, A. S; Shingare, M. S. Indian J. Chem. 2001, 408, 339.

2. Mesropyan, E. G; Ambartsumyan, G. S. Russ. J. Org. Chem. 2001, 37, 1476. 
3. Bouhfid, R.; Joly, N.; Ohmani, F.; Essassi, E. M.; Massoui, M.; Martin, P. Lett. Org. Chem. 2008, 3.

4. Pandeya, S. N.; Sriram, D.; Nath, G.; De Clercq, E. Sci. Pharm. 1999, 67, 103.

5. Ravichandran, V.; Mohan, S.; Kumar, K. S. Arkivoc 2007, (xiv), 51.

6. Terzioğlu , N.; Karalı, N.; Gürsoy, A.; Pannecouque, C.; Leysen, P.; Paeshuyse, J.; Neyts, J.; De Clercq, E. Arkivoc 2006, (i), 109.

7. Pandeya, S. N.; Sriram, D.; Nath, G.; De Clercq, E. Eur. J. Med. Chem. 2000, 35, 249.

8. Pandeya, S. N.; Sriram, D.; Nath, G.; De Clercq, E. Arzneil,-Forschun./Drug Res. 2000, 50, 55.

9. Matesic, L.; Lock, J. M.; Bremner, J. B.; Pyne, S. G.; Skropeta, D.; Ranson, M.; Vine, K. L. Bioorg. Med. Chem. 2008, 16, 3118.

10. Bhattacharya, S. K.; Glover, V.; McIntyre, I.; Oxenkrug, G.; Sandler, M. Neurosci. Lett. 1982, 92, 218.

11. Bhattacharya, S. K.; Mitra, S. K.; Acharya, S. B. J. Psychopharmacol. 1991, 5, 202.

12. Bhattacharya, S. K.; Clow, A.; Przyborowska, A.; Halket, J.; Glover, V.; Sandler, M. Neurosci. Lett. 1991, 132, 44.

13. Grabley, S.; Kluge, H.; Hoppe, H-U. Angew. Chem., Int. Ed. Engl. 1987, 99, 692.

14. Jegham, S.; Puech, F.; Burnier, P.; Berthon, D.; Leclerc, O. PCT FR 01511, 1996.

15. Kakeya, H.; Morishita, M.; Kobinata, K.; Osono, M.; Ishizuka, M.; Osada, H. J. Antibiot. 1998, 51, 1126.

16. Hutchinson, D; Brickner, S. J.; Barbachyn, M. R., Gammill, R.; Patel, M. PCT US 03570, 1993.

17. Pridgen, L. N.; Prol Jr., J. J. Org. Chem. 1989, 54, 3231.

18. Correa, A.; Denis, J.-N.; Greene, A. E. Synth. Commun. 1991, 21, 1.

19. Sudharshan, M.; Hultin, P. G. Synlett 1997, 171.

20. Wu, Y.; Shen, X. Tetrahedron: Asymmetry 2000, 11, 4359.

21. Bouhfid, R.; Joly, N.; Massoui, M.; Cecchelli, R.; Lequart, V.; Martin, P.; Essassi, E. M. Heterocycles 2005, 56, 2949.

22. Robeyns, K.; Rohand, T.; Bouhfid, R.; Essassi, E. M.; Van Meervelt, L. Acta Cryst., 2007, E63, o1747.

23. Crystallographic data can be obtained free of charge via www.ccdc.cam.ac.uk/data_request/cif, or by emailing data_request@ccdc.cam.ac.uk, or by contacting The Cambridge Crystallographic Data Center, 12, Union Road, Cambridge CB2 1EZ, UK; fax: +44 1223336033.

24. Soukri, M.; Guillaumet, G.; Besson, T.; Aziane, D.; Aadil, M.; Essassi, E. M.; Akssira, M. Tetrahedron Lett. 2000, 41, 5857. 